\title{
PolyADP-Ribosylation in Postfertilization and Genome Reprogramming: Implications for Carcinogenesis
}

\author{
Tomoharu Osada and Mitsuko Masutani \\ Additional information is available at the end of the chapter
}

http://dx.doi.org/10.5772/46097

\section{Introduction}

Pottranslational modification of proteins (PTM) is involved in molecular targeting or signal transduction as a basis of a variety of biological processes of the cells. Approximately 300 PTMs are existed in the cells, some of which are supposed to have important roles in cell physiology or embryogenesis. In addition, some modifications are reciprocally interacted, which regulates gene expression or protein dynamics or processing. We focus here polyADP-ribosylation (PARylation), which is found to contribute importantly at fertilization and postfertilization development. Moreover, we discuss the possible drug discovery of ADP-ribosylation inhibitors in carcinogenesis or fertility control.

Upon fertilization, two kinds of genetic materials meet together to generate a new organism [1]. Meanwhile, the organism develops without major expression of genes from genomes. Although maternal proteins are supposed essential for the period, the details are ill understood. Transition of zygotic gene expression is also essential for further development. Some PTMs on transcriptional factors may be key regulation to express the genes which supports early development. Parental DNAs are differently regulated in the postfertilized eggs, although the biological significance is controversial. Theses implications suggest importance of PTMs on postfertilization and transition of zygotic development. Recently we found that PARylation, a PTM of protein is important for postfertilization development, which seems similar with those of carcinogenesis.

The PARylation reaction is a PTM of proteins, which is synthesized with poly(ADP-ribose) polymerase (PARP) and metabolized with poly(ADP-ribose) glyceraldehyde (PARG) [2,3]. The reciprocal regulation is speculated as a key mechanism that underlies reversible 
regulation of gene expression. The poly(ADP-ribose) is generated from NAD, an energy reservoir of the cells by PARP. The PARP synthesizes poly(ADP-ribose) using NAD. PARP polymerizes ADP-ribose residues to generate poly(ADP-ribose) chains onto proteins. PARylation reaction itself is regulated by auto-PARylation of Parp1. Since the ADP-ribose residue is negatively charged, the acceptor proteins become negatively charged by the addition of ADP-ribose residues. The electrical charge-shift of the protein induced by PARylation may decrease an easy access of proteins into the DNA structure or may induce structural instability of protein-DNA interactions, because the negative charge of poly(ADPribose) supports its association with DNA-binding proteins, which are positively charged. The recognition of DNA sequences by protein structure may also be affected by PARylation. Upon postfertilization development, zygotic gene expression is essentially activated prior to the 4-cell cleavage stage in mice and human. Therefore, behavior of maternal molecules plays important roles at postfertilization development before zygotic gene activation. PTM of protein is supposed to contribute to protein dynamics at postfertilization development. Pharmacological blockage of PARylation revealed defects of postfertilization development in mice [4]. The data raise a question regarding the roles of PARylation at fertilization development. A wealth of study revealed that NAD is rich in eggs and rapidly degraded upon fertilization in Xenopus larvae. Parp activation at fertilization may contribute to consumption of NAD, which may bring a plausible explanation towards the uncovered subject. Postfertilization development is specific biological window to highlight the significance of PTM, because transcription is minor mechanisms for the organisms at the period. In this chapter, we discuss the roles of PARylation in eggs and the function of postfertilization development. Further we discuss the possible implications to drug discovery focusing on PARylation regulation. We focus on polyADP-ribosylation because we previously showed that no fertilized eggs were obtained by pharmacological blockage of PARylation by a PARP inhibitor, PJ-34 [4]. Other inhibitors of PARylation showed similar results. Therefore, a hypothesis was raised that posttranslational regulation is key regulation of maternal genetic materials at fertilization because no robust transcription is occurred at the beginning of life.

\section{PARylation in postfertilization development}

Recent research showed that pharmacological blockage of PARylation leads to defects in pronuclear fusion during postfertilization in mice. Based on the observations, several approaches were achieved to investigate the roles of PARylation in vivo and mechanisms of reprogramming applicable for regenerative medicines or elucidation of human diseases including carcinogenesis.

Based on our observations regarding the disorganized microtubule assembly in oocytes by PARylation inhibitors, polyADP-ribosylated proteins of oocytes and postfertilized eggs were searched. We found that the tubulins $(\alpha 1 c, \beta 2 c)[4]$ and glutathione $S$-transferees $\mu 5$ (GST $\mu 5)$ (Osada et al., unpublished data) was highly polyADP-ribosylated after fertilization. 
Because PARylation is known as a regulatory machinery of DNA surveillance system, this data provided us with a novel sight of view on the roles of PARylation regarding cell signaling and metabolisms. As a major upstream signaling cascade after sperm entry, MAPkinase (MAPK) signaling has been postulated as the key regulatory mechanism for pronucleus formation, and Parp1 is reported to be involved in the regulation of MAPK signaling [5]. Our data suggest that Parp1 is a novel nuclear component for PPN (Pseudo pronuclei) formation, which may be mediated by MAPK signaling. Phosphorylation level of Erk was decreased in the MII phase of Parp-1 null oocyte. Because the downregulation of Erk phosphorylation is known to disturb microtubule organization, these results raise a possibility that the transient administration of a Parp inhibitor may block the early phase of post-fertilization process.

Second, we focused on the roles of polyADP-ribosylation on epigenetic regulation. Accumulating study suggests that PARylation has a fundamental role in transcription as well as DNA surveillance, which mediates topological changes of the DNA structure [6,7]. We first examined the effects of PARylation on chromatin modification during postfertilization development. An immunofluorescence study using histone acetylation and methylation antibodies were initially carried out. Upregulation of dimethyl-H3k4 in the PARylation-inhibited embryos at $6 \mathrm{hpf}$, and downregulation of dimethyl-H3K9 and dimethyl-H3K27 at $15 \mathrm{hpf}$ were observed (unpublished data, Osada et al.). Recently, functional links between histone methylation and DNA methylation have been elucidated. Based on our observations of distinct effects of PARylation inhibition on histone modification regulation, we next sought for the roles of PARylation in DNA methylation. Global methylation of the maternal genome is maintained and sperm DNA is quickly demethylated during the postfertilization period [8]. The low immunoreactivity to the MetC antibody in the PARylation inhibited embryos was observed and it persisted at least until 15 hpf (unpublished data, Osada et al.). The data suggest that epigenetic regulation is asymmetrically activated during postfertilization development, although gene expression is not activated. Therefore, PARylation affects the integrity of epigenetic regulation of oocytes and postfertilized eggs (Figure 1).

An additional implication of this finding is that PARylation regulates the epigenetic nonequivalence of the pronuclei in mice. The molecular mechanisms regulating the asymmetric DNA methylation of one-cell embryos are largely unknown, and we showed that PARylation is involved in the epigenetic regulation of the mouse early development. PGC7/Stella-deficient eggs show similar defects in the protection of female pronuclei from DNA demethylation [9]. The nucleo-cytoplasmic transport of the responsible proteins appears to be important for the regulation of DNA methylation. The role of PARylation in DNA methyltransferase (DMNT) regulation is yet to be fully elucidated, although the interaction of DNMT1 with PARP1 and the indirect repression of DNMT1 activity by interacting with PAR have been suggested [10]. Based on our data showing an association between PARylation or PARP and histone modification, it is speculated that PARylation 
may regulate the accessibility of DNMT or demethylase to DNA, in a manner mediated by chromatin remodeling. Our study provides a novel avenue for better understanding of establishment of chromatin organization through the histone codes and DNA methylation that may underlie at the beginning of zygotic development.

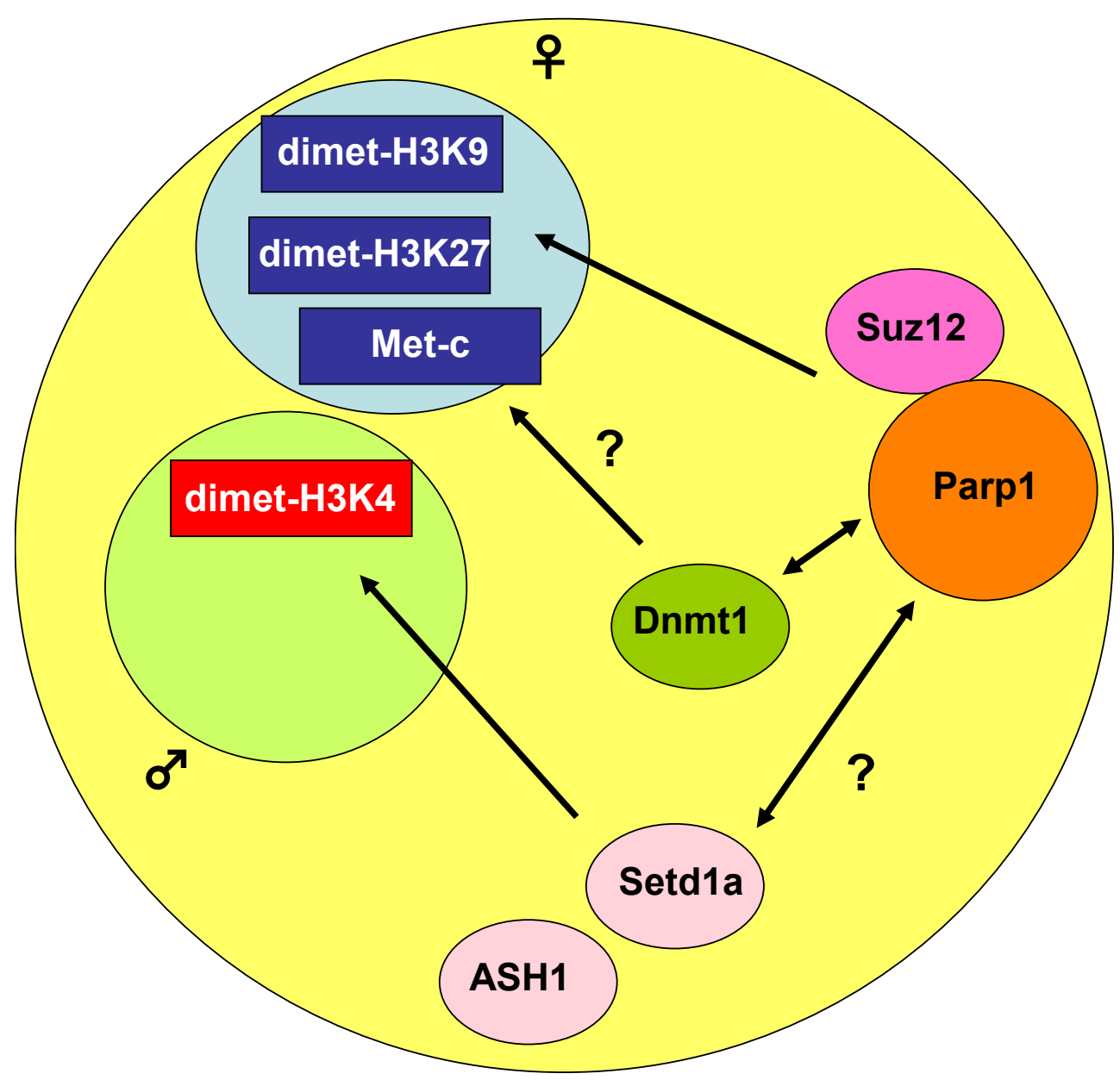

Figure 1. Scheme of putative roles of PARylation in the chromatin dynamics of one-cell embryos. In the one-cell embryos (yellow circle), PARP1 may interact with histone modifying enzymes including Suz12, that might affect the asymmetric regulation of histone modifications in the pronuclei (male pronucleus in blue circle and female pronucleus in green). 
To examine whether there is a direct or indirect linkage of epigenetic regulation with PARylation, we screened molecules that interacts with Parp1. To elucidate the molecular basis of the effect of PARylation on histone modification, we performed yeast two-hybrid screening using the bait vector carrying N-terminal and automodification domain. The Suz12, a histone methyltransferase, was identified as a candidate molecule interacting with Parp1 (unpublished data, Osada et al.). Suz12 is a component of PRC2/3/4 (Polycomb Repressive Complex 2/3/4), which regulates the repressive status of transcription by the methylation of histone H3K9 and H3K27. Our findings support the idea that Parp1 acts as a regulatory scaffold for the access of PRC2/3 to target DNA, because none of the components of the PRC2/3/4 complexes are DNA binding proteins. OCT4, bidirectional transcriptional regulator, is suggested to play a role of transcriptional regulation by recruiting $\mathrm{PRC} 2 / 3 / 4$ to the target genes, which are indispensable for the embryonic development. Modification of PARylation triggers loosening of chromatin structure, which may enable the access of transcriptional factors to DNA duplex structure.

Third, as shown in Figure 2, defects in pronuclear formation by inhibition of PARylation raise a possibility that PARylation is involved in laminar formation of pronuclei [4]. In fact, $\mathrm{HP1}$, an anchor protein of lamina and heterochromatin is polyADP-ribosylated [11]. Our findings showed prolonged presence of lamin-A/C, a core protein of lamina by PARP inhibitor PJ-34 treated eggs. Interestingly, lamin-A/C is predominantly existed in undifferentiated cells. Lamin-A/C is known as a marker of carcinogenesis [4]. Our observation provided a new insight into regulatory machinery of laminar formation in pluripotent cells by PARylation. Our initial data suggest that cell-cycle is not disturbed when embryos were subjected to treatment of PARP inhibitor.

During the first cell cycle of mouse embryos, a few genes are transcribed mainly from paternal genome. Inhibition of transcription during one-cell embryos by RNA polymerase inhibitors showed dispensable roles of transcription in normal development. Zygotic gene activation is required for progression from 2-cell to 4-cell embryos. These indicate that posttranslational regulation of protein should act as a stem mechanism of the development of one-cell embryos. Upon fertilization, highly compacted chromatin of gametes was acutely decondensed to form pronuclei $(\mathrm{PN})$ within a few hours. Protamines of sperm chromatin are replaced by maternal histone $\mathrm{H} 1$ during this process, which may be associated with global hypomethylation of sperm-derived PN. In contrast, maternal chromatin arrested at metaphase II progresses rapidly into G1 phase and subsequently forms the female PN. DNA synthesis from paternal genome is preceded to that from maternal DNA. A minor transcription is activated solely from male PN. This evidence suggests that the requirement for the posttranslational regulations of parental genomes before mingling of both gamete DNA to begin the proper zygotic development. Of posttranslational modification of proteins, we examined here the effects of PARylation during the first cell cycle of mouse embryos. Metabolism of NAD, which is the substrate of PARPs, is acutely activated upon fertilization. Further analysis will elucidate the biological functions of PARylation upon fertilization and downstream target molecules of them. 


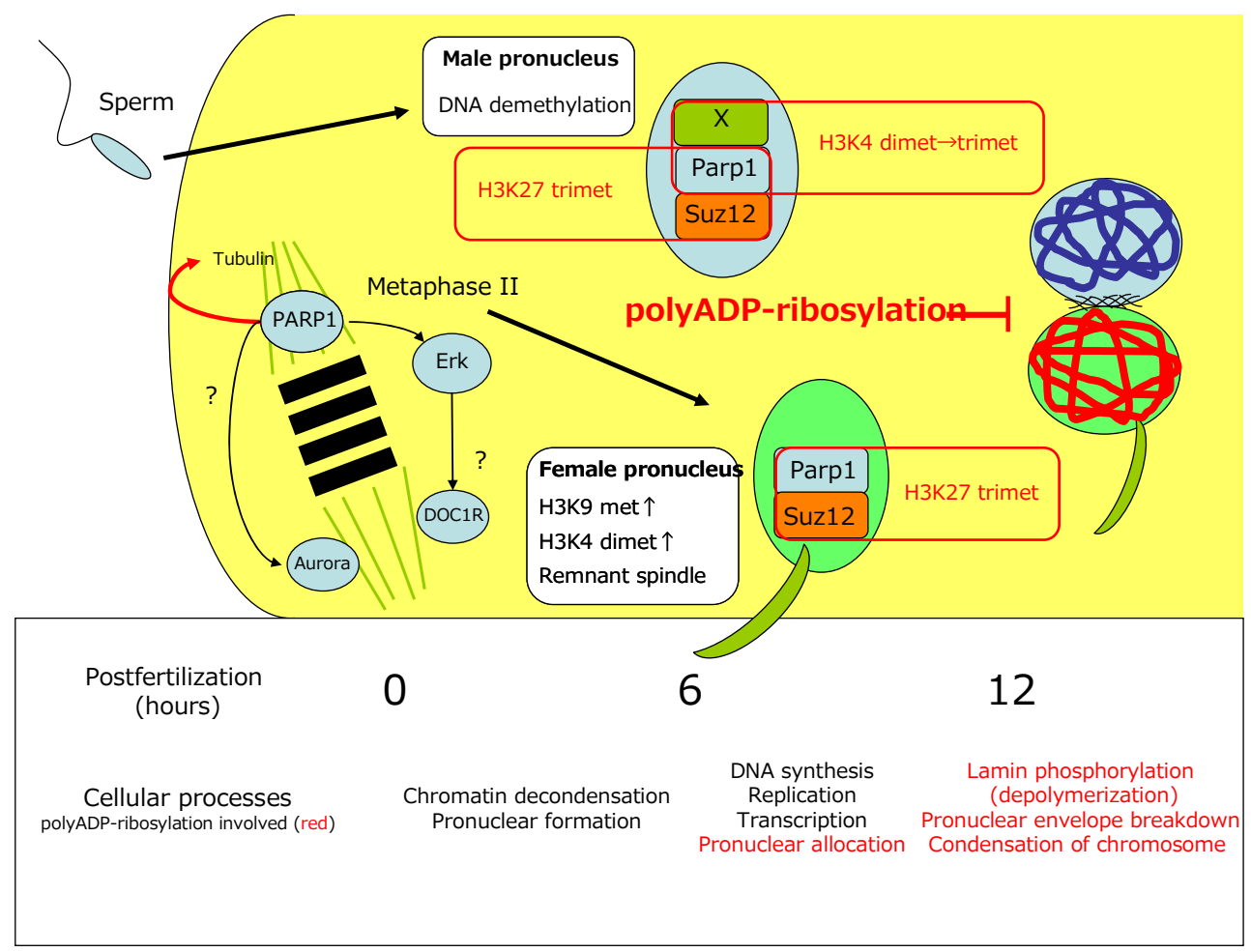

Figure 2. Model for regulation of pre- and postfertilization process by PARylation. Biological processes occurred during pre- and postfertilization were shown. Putative correlation with polyADPribosylation were indicated in red. 


\section{Implication towards reprogramming}

In 1997, the first cloned animals were generated [12]. Cytogenetic analyses have shown that karyotypic aberrations occur in cloned embryos during the first mitotic cleavage. Epigenetic errors in cloned animals are also argued to be the major reason for the limited success rates of cloned animal births. However, whether genome-wide chromatin remodeling during nuclear reprogramming causes DNA damage, or whether defects in DNA repair cause the inefficiency of cloned animal births have not been investigated. Recently, it was reported that double strand break (DSB)-mediated chromatin remodeling regulates transcription, and that Parp1 is critically involved in this process. Various roles for Parp1 have been described, including in the chromatin remodeling involved in transcriptional regulation [2,3]. The roles of Parp1 in NT-embryo development in the contexts of DNA repair and chromatin remodeling was examined. To do this, we used Parp1-null mutant cells as recipient oocytes and as a source of donor nuclei.

We observed that the activation process in NT eggs was enhanced in Parp1-null NT embryos, although some genomic instability was observed (Osada T., unpublished data). Dynamic changes in histone acetylation and methylation were induced under Parp-1 deficiency in NT embryos (Figure 3). The lack of Parp1 may thus facilitate chromatin condensation or transcriptional silencing. Parp1 is involved in both the regulation of DNA strand break repair and the epigenetic control of gene expression. Our findings suggest that Parp1 is important for chromatin remodeling possibly through histone modification during the nuclear reprogramming of NT embryos. The phosphorylation of histone H2AX at DSBs is believed to be crucial for the recognition and repair of DNA damage. The foci-like presence of $\gamma \mathrm{H} 2 \mathrm{AX}$ in the NT embryos implied that DNA repair was taking place in the PNN. H2AX is a direct target of ATM kinase, which is expressed at the spindle of MII oocytes, and Parp1 interacts with ATM. The prolongation of the $\gamma \mathrm{H} 2 \mathrm{AX}$ foci in the Parp $1^{-1-}$ NT embryos could therefore be related to a delay in repairing DNA strand breaks, as is the case with Parp $1^{-/}$MEF and ES cells, which show prolonged $\gamma \mathrm{H} 2 \mathrm{AX}$ foci after DNA damage induced by neocartinostatin. In Parp $1^{--}$NT embryos, mitotic arrest of embryogenesis at the 2-4-cell stage was frequently observed, accompanied by polynucleated blastomeres. Since Parp1 is located in centrosomes as well as in nuclei, the lack of Parp1 may have disturbed the normal cell division cycle and the synchronous pattern of cell division among the blastomeres. Transient Parp-1 functional inhibition may be therefore useful to improve the efficiency of NT by modulating the dynamic organization of chromatin without causing genomic instability.

\section{Implication towards human diseases}

PARylation has been known to possess important roles in carcinogenesis. PARP mainly affects DNA surveillance system in the cells, which deficit increases the risk of carcinogenesis. In addition, recent study showed a functional relationship between PARP 
and MAPK signaling, known as a major intracellular signaling of transformation into cancer cells. Although morphological change of nuclear envelope has been known in cancer cells, the molecular mechanism of the processes is not well understood. Recent studies revealed molecular signaling of laminar formation is altered during carcinogenesis. Our study revealed that PARylation is involved in these molecular events. Oocytes are capable of being isolated without other cell types by adding hyarulonidase, which is far easier compared with the difficulty in homogenous isolation of purified cancer cells. Our data suggest oocytes provide with a unique biological window for elucidating the mechanism of PARylation in carcinogenesis. Further comparative analysis between cancer cells and oocytes may highlight the uncovered mechanisms underlying the carcinogenesis processes.

\section{$\mathrm{PARP}^{+/+}$cloned one-cell embryo}
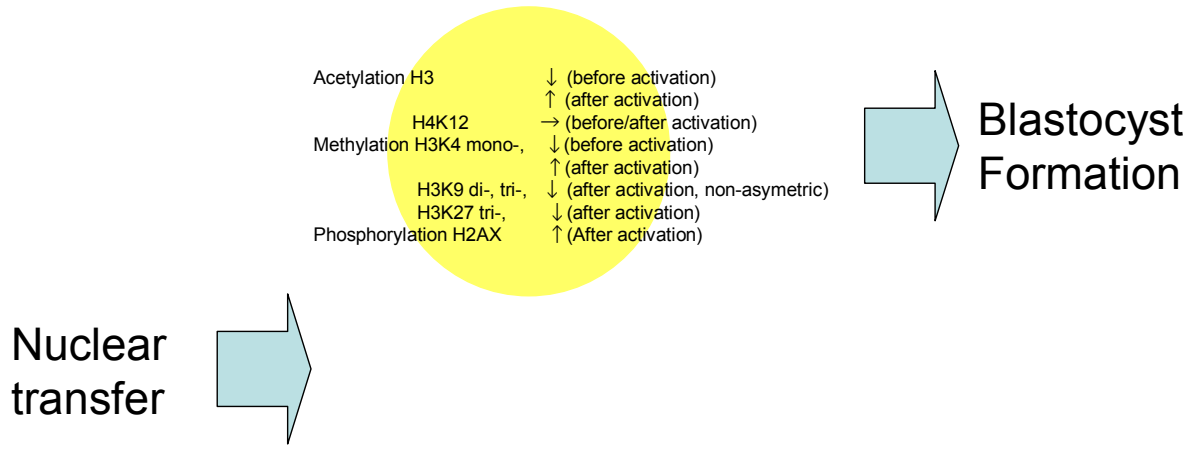

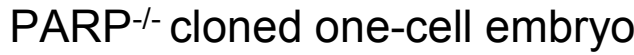
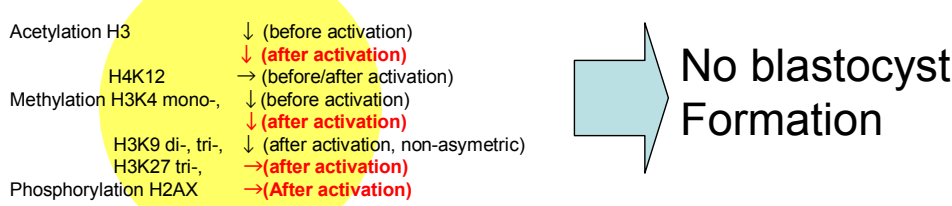

Figure 3. The scheme of histone modification and the effects of Parp1 deficiency during the first cleavage of NT embryogenesis. Histone modifications of the one-cell reconstructed oocytes were indicated in $\mathrm{Parp}^{+/}$, and those which Parp1 deficiency influences were indicated in Parp $-1-$ in red. 
We also described here that PARylation is a novel target of anti-conception. Inhibition of PARylation may easy to be handled, because exposure of an inhibitor to oocytes is effective enough to stop fertilization. Further extensive analysis should be carried out regarding the optimized dose of inhibitors and non-toxic dose on other tissues. Biological phenomena, in which PARylation is involved, are variable and its inhibitors could serve as possible pharmaceutical targets including inflammation or brain injury as well as carcinogenesis and reproduction as discussed in this chapter. Further basic investigation of the roles of PARylation in the cells will broaden the view for the understanding the embryogenesis and proof of mechanism of human diseases for drug discovery.

\section{Author details}

Tomoharu Osada and Mitsuko Masutani

Mitsubishi Chemical Medience Corporation, Kashima Laboratory, Japan

Division of Genome Stability Research, National Cancer Center Research Institute, Japan

\section{Acknowledgement}

We thank Dr. Ryuzo Yanagimachi and Dr. Takashi Sugimura for giving informative insight throughout the study. This study is partly supported by a Grand-in Aid for the Third Comprehensive 10-Year Strategy for Cancer Control from the Ministry of Health, Labor and Welfare of Japan.

\section{References}

[1] Yanagimachi, R (1994) Mammalian fertilization. In: Knobil E, Neil JD, editors. Physiology of Reproduction. $2^{\text {nd }}$ edition. New York: Raven Press; pp. 189-317.

[2] Miwa, M. and Masutani (2007) M. PolyADP-ribosylation and cancer. Cancer Sci. 98:1528-1535.

[3] Schreiber V, Dantzer F, Ame JC, de Murcia G (2006) Poly(ADP-ribose): novel functions for an old molecule. Nat Rev Mol Cell Biol. 7: 517-528.

[4] Osada T, Ogino H, Hino T, Ichinose S, Nakamura K, Omori A, et al. (2010) PolyADPribosylation is required for pronuclear fusion during postfertilization in mice. PLoS One 5:e12526. doi:10.1371/journal.pone.0012526

[5] Cohen-Armon M, Visochek L, Rozensal D, Kalal A, Geistrikh I (2007) DNAindependent PARP-1 activation by phosphorylated ERK2 increases Elk1 activity: a link to histone acetylation. Mol. Cell. 25:297-308.

[6] Tulin A, Spradling A (2003) Chromatin loosening by poly(ADP)-ribose polymerase (PARP) at Drosophila puff loci. Science 299: 560-562.

[7] Caiafa P, Guastafierro T, Zampieri M (2009) Epigenetics: poly(ADP-ribosyl)ation of PARP-1 regulates genomic methylation patterns. FASEB J 23: 672-678.

[8] Mayer W, Niveleau A, Walter J, Fundele R, Haaf T (2000) Demethylation of the zygotic paternal genome. Nature 402: 501-502. 
[9] Nakamura T, Arai Y, Umehara H, Masuhara M, Kimura T, et al (2007) PGC/Stella protects against demethylation in early embryogenesis. Nat. Cell Biol. 9: 64-71.

[10] Reale A, Matteis GD, Galleazzi G, Zampieri M, Caiafa P, 2005 Modulation of DNMT1 activity by ADP-ribosylation. Oncogene 24: 13-19.

[11] Quenet D, Gasser V, Fouillen L, Cammas F, Sanglier-Cianferani S, et al (2008) The histone subcode: poly(ADP-ribose) polymerase-1 (Parp-1) and Parp-2 control cell differentiation by regulating the transfcriptional intermediary factor TIF beta and heterochromatin protein HP1alpha. FASEB J. 22: 3853-3865.

[12] Wilmut I, Schnieke AE, McWhir J, King AJ. Campbell KH (1997) Viable offspring derived from fetal and adult mammalian cells. Nature 385:810-813. 